\section{(6) OPEN ACCESS}

\title{
Ten years transmission of the new variant of Chlamydia trachomatis in Sweden: prevalence of infections and associated complications
}

\author{
Jenny Dahlberg, ${ }^{1}$ Ronza Hadad ${ }^{2}$ Karin Elfving, ${ }^{3}$ Inger Larsson, ${ }^{4}$ Jenny Isaksson, ${ }^{1}$ \\ Anders Magnuson, ${ }^{5}$ Hans Fredlund, ${ }^{2}$ Magnus Unemo, ${ }^{2}$ Björn Herrmann ${ }^{1}$
}

\begin{abstract}
- Additional material is published online only. To view please visit the journal online (http://dx.doi.org/10.1136/ sextrans-2016-052992)
\end{abstract}

${ }^{1}$ Section of Clinical Bacteriology, Department of Medical Sciences, Uppsala University, Uppsala, Sweden

${ }^{2}$ WHO Collaborating Centre for Gonorrhoea and other STIS, Örebro University, Örebro, Sweden

${ }^{3}$ Department of Clinical Microbiology, Falu Lasarett, Falun, Sweden

${ }^{4}$ Department of Clinical

Microbiology, Sunderby Hospital, Luleå, Sweden

${ }^{5}$ Department of Clinical Epidemiology and Biostatistics, School of Medical Sciences, Örebro University, Örebro, Sweden

Correspondence to Dr Bjőrn Herrmann, Section of Clinical Bacteriology, Department of Medical Sciences, Uppsala University, SE-75185 Uppsala, Sweden; bjorn. herrmann@medsci.uu.se

Received 18 November 2016 Revised 21 April 2017 Accepted 19 May 2017 Published Online First 19 July 2017
Check for updates

To cite: Dahlberg J, Hadad R, Elfving K, et al. Sex Transm Infect 2018:94:100-104

\section{ABSTRACT \\ Objectives In 2006, a new variant of Chlamydia} trachomatis (nvCT) was discovered in Sweden. It has a deletion in the plasmid resulting in failed detection by the single target systems from Abbott and Roche used at that time, whereas the third system used, from Becton Dickinson (BD), detects nvCT. The proportion of nvCT was initially up to $65 \%$ in counties using Abbott/Roche systems. This study analysed the proportion of nvCT from 2007 to 2015 in four selected counties and its impact on chlamydia-associated complications.

Methods C. trachomatis-positive specimens collected from 2007 to 2015 were analysed by a specific PCR to identify nvCT cases. Genotyping was performed by multilocus sequence typing (MLST) and ompA sequencing. Ectopic pregnancy and pelvic inflammatory disease records were extracted from the national registers.

Results In total, 5101 C. trachomatis-positive samples were analysed. The nvCT proportion significantly decreased in the two counties using Roche systems, from $56 \%$ in 2007 to $6.5 \%$ in 2015 ( $p<0.001)$. In the two counties using BD systems, a decrease was also seen, from $19 \%$ in 2007 to $5.2 \%$ in 2015 ( $p<0.001$ ). Fifteen nvCT cases from 2015 and 102 cases from 2006 to 2009 had identical MLST profiles. Counties using Roche/Abbott systems showed higher mean rates of ectopic pregnancy and pelvic inflammatory disease compared with counties using BD systems.

Conclusions The nvCT proportion has decreased in all counties and converged to a low prevalence irrespective of previous rates. Genotyping showed that nvCT is clonal and genetically stable. Failing detection only marginally affected complication rates.

\section{INTRODUCTION}

A new variant of Chlamydia trachomatis (nvCT) was discovered in Sweden in 2006. ${ }^{1}$ nvCT has a deletion in its plasmid that included the target sequences used by two diagnostic single target nucleic acid amplification tests (NAATs) at that time, Abbott m2000 (Abbott Laboratories, Des Plaines, Illinois, USA) and Amplicor/COBAS Amplicor/TaqMan48 (Roche, Molecular Systems, Branchburg, New Jersey, USA). The proportion of nvCT was between $20 \%$ and $65 \%$ in counties using these Abbott/Roche NAATs in $2006 .^{2}$ Retrospective analysis showed nvCT was present already in
$2003^{3}$ and a conservative calculation estimates the number of false-negative tests to 15000 in Sweden from 2003 to 2006, that is, when nvCT was undetected. Abbott and Roche subsequently designed dual-target assays that could detect also nvCT. The third NAAT used in Sweden at that time was ProbeTec from Becton Dickinson (BD, Franklin Lakes, New Jersey, USA). This NAAT has always been able to detect nvCT because it uses another target region on the plasmid. The proportion of nvCT in counties using the BD NAAT ranged from $7 \%$ to $19 \%$ in $2006-2007 .^{2}$

The objectives of the present study were (1) to determine the current nvCT prevalence (2015) and relate it to the prevalence in previous years; (2) to analyse if the nvCT is of clonal nature; and (3) to investigate available records of chlamydia-associated complications between 2000 and 2014 and associate it with the nvCT prevalence and diagnostic methods used.

\section{MATERIALS AND METHODS}

\section{Study populations and detection methods}

Two counties using Roche NAAT for detection of C. trachomatis, and two counties using BD NAAT in 2006 were selected. The Roche counties Dalarna and Örebro counties had the highest reported nvCT rate in $2006^{2}$ and were therefore important to follow over time. The Uppsala county is a neighbour to several Abbott/Roche counties and showed the influx of nvCT into an area using the BD system, while the BD county Norrbotten is remote and had no neighbour county using Abbott/Roche diagnostics in 2006. In the two Roche counties, the COBAS TaqMan48 NAAT was replaced by ompAbased NAATs in November 2006 and January 2007, respectively. The new dual target version COBAS TaqMan CT Test v2.0, which detects nvCT, was implemented in mid-2008. The study periods in the four counties are shown in online supplementary table 1 .

Mainly consecutive C. trachomatis-positive specimens (one per patient) were collected in each county in 2007, 2008, 2009, 2011 and 2015. The time periods differed between counties from 30 to 90 days from November 2006 to April 2007. In the other years, collection periods varied from 60 to 147 days from January to July. The length of collection periods was chosen to show differences in 
nvCT proportion between counties using Roche and BD NAATs. DNA extraction methods for the years 2007-2009 have been described earlier, ${ }^{4}$ and evaluations have shown that the change of DNA extraction method in the two Roche counties did not have any considerable impact on C. trachomatis detection when compared with the Roche NAAT used until 2006.

Specific detection of nvCT and wild-type C. trachomatis was based on size differentiation in a PCR method targeting the plasmid by use of primers from a method by Ripa and Nilsson, ${ }^{5}$ as previously described. ${ }^{4}$ After exclusion of non-typeable cases, we calculated differences in proportions of nvCT with 95\% CIs and compared them using a $\chi^{2}$ test (Minitab Statistical Software V.17; Minitab, State College, Pennsylvania, USA).

Logistic regression was used to evaluate proportions of nvCT of all detected C. trachomatis cases as a relative linear trend expressed as OR per year measured in 2007, 2008, 2009, 2011 and 2015 and furthermore evaluate if the relative trends per year were different between counties using Abbott/Roche and $\mathrm{BD}$ by interaction tests. Poisson regression was used to compare rates of ectopic pregnancy and pelvic inflammatory disease (PID) between counties using Abbott/Roche and BD C. trachomatis detection systems.

\section{Genotyping}

The genetic stability of nvCT was analysed by sequence determination of $o m p A$ and five multilocus sequence typing (MLST) genes as described in the protocol available at http://mlstdb.bmc. uu.se/.

\section{Chlamydia-associated complication episodes}

The numbers of reported C. trachomatis cases in all Swedish counties were obtained from The Public Health Agency of Sweden. C. trachomatis infections are included in the Swedish Communicable Diseases Act and are mandatory to report. The statistics on PID and ectopic pregnancy from 2000 to 2014 (inpatient diagnoses) and from 2005 to 2014 (outpatient diagnoses), and number of births (including stillbirths) were obtained from The National Patient Register, The National Board of Health and Welfare (http://www.socialstyrelsen.se/statistik/statistikdatabas). All diagnoses of ectopic pregnancy (International Classification of Disease 10th Revision codes corresponding to ICD 10 O00) and PID (N70, 71, 73) were included. Data on number of women in different counties were retrieved from Statistics Sweden (http://www.scb.se/en).

\section{RESULTS}

In total, 5101 samples were analysed, of which 286 (5.6\%) were untypeable and excluded from the study. The number of samples ranged from 115 to 377 per year from each county (see online supplementary table 1). All counties showed a significant decrease in the proportion of nvCT from 2007 to 2015 expressed as OR per year; however, the nvCT proportion in the counties using Roche NAAT decreased significantly more compared with the counties using BD NAAT $(\mathrm{p}<0.01$, interaction test). In the two Roche counties combined, the proportion of nvCT was 56\% in 2007 and the decrease showed an OR of 0.71 (95\% CI 0.68 to 0.74 ) per year, resulting in an nvCT proportion of $6.5 \%$ in 2015. In the two counties using BD NAAT combined, the proportion of nvCT was $19 \%$ in 2007 and the decrease showed an OR of 0.82 (95\% CI 0.78 to 0.86 ) per year, resulting in an nvCT proportion of 5.3\% in 2015.

Separate analysis of the two BD counties showed contrasting trends. In Norrbotten, there was an initial increase from $9 \%$ in

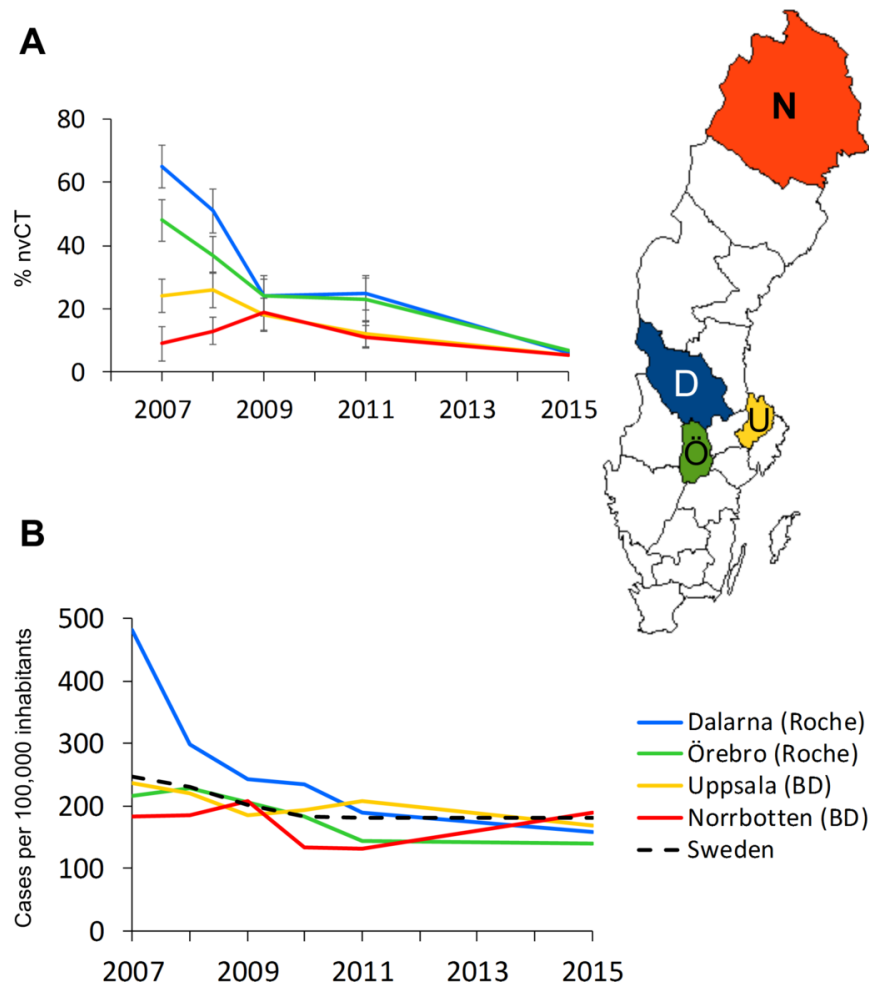

Figure 1 (A) Proportion of the nvCT of all cases positive for Chlamydia trachomatis in two counties using the Roche diagnostic system (blue/green) and two counties using the Becton Dickinson system (red/yellow). Differences were significant, with nonoverlapping 95\% Cls in 2007 and 2008. (B) Number of diagnosed cases of $C$. trachomatis per 100000 inhabitants from February to June in 2007-2011 and 2015. nvCT, new variant of Chlamydia trachomatis.

2007 to $19 \%$ in 2009 and thereafter a continuous decline in the nvCT proportion. In Uppsala, there was an overall decline from $24 \%$ in 2007 , followed by $27 \%$ in 2008 and then a continuous fall to $5.4 \%$ in 2015 . The difference in nvCT proportion between the two Roche counties and the two BD counties was obvious in 2008, but was not significant in 2009 (Roche counties 24\% (97/405); BD counties 18\% (72/391), figure 1A). In 2011, the nvCT proportion remained at 24\% (98/404) in the Roche counties but declined to $11 \%(64 / 559)$ in the BD counties; this difference of $13 \%$ was significant ( $95 \%$ CI $11,15 \%, \mathrm{p}<0.001$ ). In 2015 , the difference had decreased to $1.3 \%$ (Roche counties $6.5 \%$ (43/666); BD counties 5.2\% (37/697)). Detailed information is given in online supplementary table $2 \mathrm{~A}$.

The proportion of nvCT was compared by age (see online supplementary table $2 \mathrm{~B}$ ). The individuals infected with nvCT were initially younger in counties using Roche NAAT, both in 2007 and 2008, but not in 2009 and 2011 (data not shown). In 2015, the proportion of nvCT was significantly higher in the age group $\geq 30$ years in the Roche counties $(p<0.001)$. In counties using BD NAAT, nvCT was not significantly associated with age group at any time point. No difference in the proportion of nvCT was seen between the genders (data not shown).

The total number of C. trachomatis cases per 100000 inhabitants, diagnosed from January 2007 to June 2007, was higher in Dalarna than in the other counties (figure 1B). The incidence in Dalarna declined dramatically in 2008; and thereafter the differences between the four counties were reduced and were not associated with diagnostic method. National C. trachomatis rates show that since 1997, the number of diagnostic tests used 


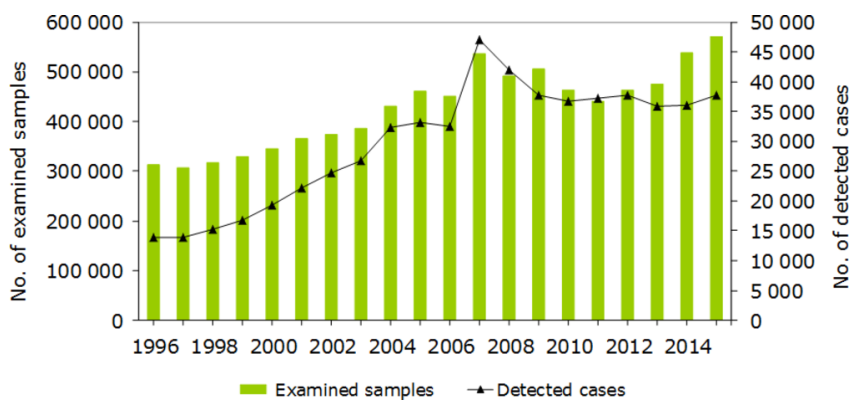

Figure 2 Chlamydia trachomatis reports, Sweden, 1996-2015. Since 1997, the number of individuals examined and cases detected increased. The figures for 2005 and 2006 reflect the failure to detect cases of the nvCT in 13 of 21 counties. In 2007, when C. trachomatis nucleic acid amplification tests detecting nvCT were implemented, a transient increase was seen. nvCT, new variant of Chlamydia trachomatis.

and cases detected increased (figure 2). The number of detected cases in $2005(n=33162)$ and $2006(n=32513)$ was substantially affected by the failure to detect nvCT. Thereafter, a transient increase was seen in $2007(\mathrm{n}=47103)$, when C. trachomatis NAATs detecting nvCT were implemented. In recent years, the C. trachomatis rates have levelled off and nvCT has no visible impact on national statistics.

The genetic stability was analysed and $15 \mathrm{nvCT}$ cases from 2015 (6 from Roche counties; 9 from BD counties) were identical in the 5 MLST genes as well as in ompA.

The number of reported ectopic pregnancies per 1000 births in the age group 15-39 years differed when the 13 counties using Abbott or Roche NAATs in 2006 were compared with the eight counties using the BD NAAT for $C$. trachomatis detection (figure 3A). During the years 2000-2014, the hospitalised mean rate was $1.06(95 \%$ CI 1.03 to 1.09$)$ times higher in counties using Abbott/Roche compared with counties using BD, and more pronounced in the first 8 years (2000-2007), relative risk (RR) 1.09 (95\% CI 1.05 to 1.13), than the latter years, RR 1.02 (95\% CI 0.98 to 1.07). The outpatients mean rate was also significantly higher in the years 2005-2014, RR 1.12 (95\% CI 1.08 to 1.17). The mean rate of the hospitalised PID/100 000 women aged 15-39 years was 1.03 (95\% CI 1.00 to 1.06$)$ and the outpatient mean rate was 1.10 (95\% CI 1.07 to 1.13) times higher in Abbott/Roche counties compared with BD counties (figure 3B).

\section{DISCUSSION}

The proportion of nvCT in 2006-2007 was reported to be considerably higher in counties using Abbott/Roche NAATs than in counties using BD NAAT. ${ }^{2}$ The present study shows that the nvCT proportion rapidly decreased in Roche counties after re-establishment of NAATs detecting nvCT. In the following years, the proportions of nvCT converged in all four counties and are now on the same low level. Genotyping showed that the nvCT emergence was of clonal nature. Despite severe failures in C. trachomatis detection capacity for some years, the appearance of nvCT had a statistically significant but limited effect on the rates of chlamydia-associated complications.

A main strength of our study is its design allowing the nvCT rates to be followed in selected counties in Sweden over a long time period. As briefly mentioned above, the Dalarna and Örebro counties had the highest reported nvCT rate in $2006^{2}$ and were therefore important to follow over time. The Uppsala county is a neighbour to several Abbott/Roche counties and showed the influx of nvCT into an area using the $\mathrm{BD}$ system, while the Norrbotten county is remote and had no neighbour county using Abbott/Roche diagnostics in 2006. Limitations in the present study are the relatively low number of analysed samples in the individual counties and in specific age groups, as well as that our studied counties might not in detail reflect the situation in all Swedish counties. Population movements between counties may also affect the representativeness of the numbers in the study. However, for the 15-44 year age group only $4 \%$ moved between Swedish counties in any of the study years. Thus, this limitation had no impact on the main conclusion of decreasing and converging nvCT rates.

The decreasing nvCT rates are not surprising considering the loss of the selective diagnostic advantage when appropriate detection methods were reintroduced. This is also supported by the unaltered biological fitness reported for nvCT. ${ }^{6}$ The emergence of nvCT created Sweden into a natural experiment to see what happen when opportunistic chlamydia screening was 'stopped.' Obviously, chlamydia infections were increasingly spread when testing failed and subsequent treatment and mandatory partner notification diminished. Thus, if Sweden not had its policy of widespread testing for decades, chlamydia rates would probably have been even higher. After reintroduction of adequate detection methods chlamydia rates first increased sharply and thereafter levelled off.

We have earlier shown that the spread of nvCT is of clonal nature. This is further confirmed in the present study where the
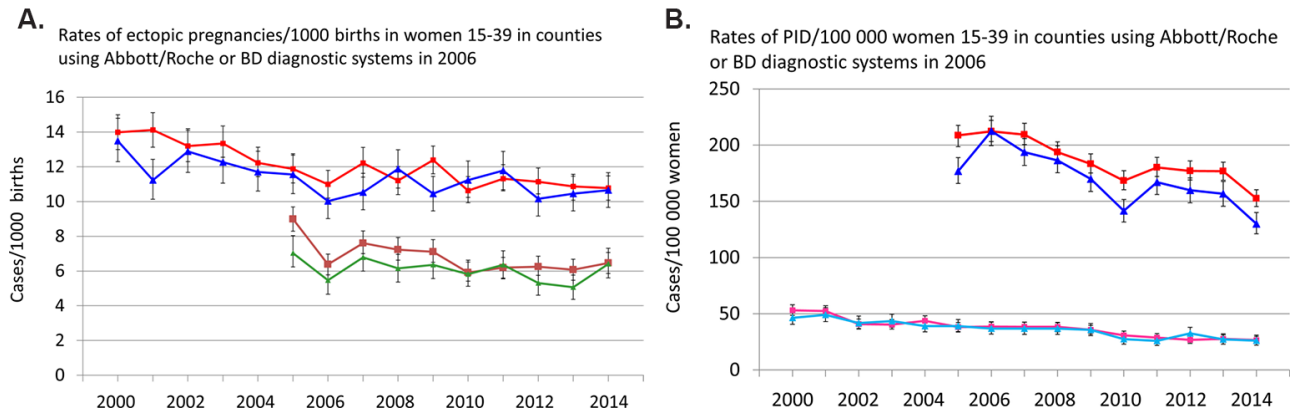

Figure 3 (A) Rates of ectopic pregnancies/1000 births in women aged 15-39years in all Swedish counties using Abbott/Roche ( $\mathrm{n}=13$; red and brown squares) or BD ( $n=8$; blue and green triangles) diagnostic systems in 2006. Hospitalised cases registered 2000-2014, and outpatient cases 2005-2014. (B) Rates of pelvic inflammatory disease/100 000 women aged 15-39years in counties using Abbott/Roche (red and magenta squares) or BD (blue and light blue triangles) diagnostic systems in 2006. Hospitalised cases registered 2000-2014, and outpatient cases 2005-2014. BD, Becton Dickinson. 
strains from 2015 were identical in the five MLST target genes with 102 nvCT strains from 2006 to $2009 .^{2-47}$ The only difference was seen in 2007 when the additionally examined gene ompA in two related cases differed by a single nucleotide polymorphism. These results also show that the MLST scheme used is sufficiently stable over time, a prerequisite for reliable genotyping. This also confirms the stability previously shown both in laboratory experiments and in previous studies of lymphogranuloma venereum. ${ }^{8}$ Whole genome sequencing would increase the resolution; however, it would not change the main conclusion regarding the clonal nature of nvCT. Accordingly, considering the cost and especially the technical challenges to obtain complete C. trachomatis genomes with high coverage from clinical samples, we did not find it essential to perform such an analysis in our study.

Except from Norway, nvCT has been reported as absent or only a few cases have been detected in studies from several European countries. ${ }^{27-14}$ This limited spread might partly be explained by the fact that sexual contacts are mostly living within a short distance and are only rarely long-distance contacts. ${ }^{15}$ Accordingly, sexual networks of risk groups for the nvCT within Sweden (mostly young heterosexual men and women) may be localised and thus only reach outside the country to a limited extent. This explanation is also supported by the fact that nvCT is a genotype $\mathrm{E}$ strain, a variant that rarely has been found in networks of men who have sex with men, which are frequently large and stretch more internationally. An additional explanation is the NAATs used in neighbouring countries. For example, in Denmark Abbott/Roche NAATs comprised about 30\% of the C. trachomatis NAATs in 2007, and substantially less in the Copenhagen area with the closest interaction with Sweden. ${ }^{16}$ Norway is the only country that has reported notable numbers of nvCT, which accounted for $3 \%$ of C. trachomatis cases detected by a BD NAAT in $2008 .{ }^{17}$ Considering that $20 \%$ of the age group $17-25$ years in the capital Oslo are Swedes, this indicates that nvCT has been imported to Norway, but it has not spread dramatically.

Considering an estimated number of 8000 false-negative C. trachomatis tests due to nvCT in $2005-2006^{18}$ or 15000 false-negative tests in 2003-2006, which are substantial proportions of all positive cases in Sweden, there is reason to suspect an elevated number of complication episodes such as PID and ectopic pregnancy, at least in counties that used Roche/ Abbott NAATs in 2006. In such counties, higher mean rates of complications were also seen compared with counties using BD NAAT. A limitation of the study is that the national register is incomplete. However, on average $87 \%$ of patient visits and linked diagnoses are reported to The National Patient Register, The National Board of Health and Welfare (Anders Jacobsson, personal communication). Therefore, the obtained figures can be assumed to mirror the real situation. Another limitation for analysis is that C. trachomatis infections are responsible for only a fraction of all complications. In recent studies, the population excess fraction of PID due to C. trachomatis infections was between $20 \%$ and $30 \%{ }^{19}$ and for ectopic pregnancy not more than $10 \%,{ }^{20}$ which decrease the possibility to see an impact of nvCT on complication rates. The time gap between chlamydia infection, with eventual PID, and the conception leading to ectopic pregnancy varies for individuals, which also affects the possibility of observing an effect of nvCT on complication rates. It would have been preferable to correlate C. trachomatis rates to complication rates directly in the four study counties, but the low number of complication episodes in the individual counties would not enable a meaningful analysis. Hence, national figures were used instead. The management of PID cases has changed over time and patients have been less often hospitalised in recent years, which also affect the number of reported cases. On the other hand, the incompleteness of reporting and the changes in management are the same in both categories of counties

A retrospective study following individuals, like a comprehensive study in Denmark, ${ }^{21}$ would increase the strength to investigate the impact of nvCT on complication rates, but it is impossible to collect the necessary data in Sweden. It would be more feasible with a modelling study of the spread of nvCT and its impact on associated complications. Altogether, the low number of registered complication episodes in the present study indicates that the risk of acquiring C. trachomatis-associated complications is lower than generally considered and is in agreement with our previous study ${ }^{22}$ and also with recent modelling studies. ${ }^{23}$ The increasing support for a low risk of complications caused by C. trachomatis infections might stress the need for new evaluations of the policy with widespread chlamydia testing as in Sweden and other countries.

In summary, nvCT spread rapidly in Sweden in the years before the discovery in 2006, but the rates decreased after changes to adequate detection methods in counties that used Abbott/Roche diagnostics at that time point. The nvCT proportion of all C. trachomatis cases has now converged to a low level irrespective of detection system used. The appearance of nvCT has only marginally affected the rates of C. trachomatis-associated complications in Sweden.

\section{Key messages}

- Due to failing detection methods, the new variant of Chlamydia trachomatis (nvCT) spread rapidly in Sweden in the years before the discovery in 2006.

- The nvCT rates have declined to a low level in 2015, irrespective of previously used detection methods.

- The C. trachomatis-associated complications were higher in counties using failing detection systems in 2006, but the spread of nvCT had only a marginal effect on the complication rates in Sweden.

\section{Handling editor Jackie A Cassell}

Contributors $\mathrm{BH}, \mathrm{HF}$ and $\mathrm{MU}$ designed the study; $\mathrm{BH}, \mathrm{KE}, \mathrm{IL}, \mathrm{HF}$ and $\mathrm{MU}$ coordinated the study and all surveillance sites; JD, JI and RH performed all the laboratory work; JD, RH and BH performed the initial data analysis; AM performed statistical analysis; JD and $\mathrm{BH}$, with support of $\mathrm{RH}, \mathrm{HF}$ and $\mathrm{MU}$, wrote the first draft of manuscript. All authors contributed to the finalisation of the manuscript.

Funding This work was supported by grants from local funds at Uppsala University Hospital and from the Örebro County Council Research Committee and the Foundation for Medical Research at Örebro University Hospital, Sweden.

Competing interests None declared.

Ethics approval The study was approved by the Regional Ethical Review Board in Uppsala, Sweden (Dnr2007/312).

Provenance and peer review Not commissioned; externally peer reviewed.

Open Access This is an Open Access article distributed in accordance with the Creative Commons Attribution Non Commercial (CC BY-NC 4.0) license, which permits others to distribute, remix, adapt, build upon this work non-commercially, and license their derivative works on different terms, provided the original work is properly cited and the use is non-commercial. See: http://creativecommons.org/ licenses/by-nc/4.0/

(c) Article author(s) (or their employer(s) unless otherwise stated in the text of the article) 2018. All rights reserved. No commercial use is permitted unless otherwise expressly granted. 


\section{REFERENCES}

1 Ripa T, Nilsson P. A variant of Chlamydia trachomatis with deletion in cryptic plasmid: implications for use of PCR diagnostic tests. Euro Surveill 2006;11:E061109.2.

2 Herrmann B, Törner A, Low N, et al. Emergence and spread of Chlamydia trachomatis variant, Sweden. Emerg Infect Dis 2008;14:1462-5.

3 Jurstrand M, Fredlund $\mathrm{H}$, Unemo M. The new variant of Chlamydia trachomatis was present as early as 2003 in Orebro County, Sweden, but remained undetected until 2006. Sex Transm Infect 2013;89:607-8.

4 Klint M, Hadad R, Christerson L, et al. Prevalence trends in Sweden for the new variant of Chlamydia trachomatis. Clin Microbiol Infect 2011;17:683-9.

5 Ripa T, Nilsson PA. A Chlamydia trachomatis strain with a 377-bp deletion in the cryptic plasmid causing false-negative nucleic acid amplification tests. Sex Transm Dis 2007;34:255-6.

6 Unemo M, Seth-Smith HM, Cutcliffe LT, et al. The Swedish new variant of Chlamydia trachomatis: genome sequence, morphology, cell tropism and phenotypic characterization. Microbiology 2010;156:1394-404.

7 Unemo M, Olcén P, Agné-Stadling I, et al. Experiences with the new genetic variant of Chlamydia trachomatis in Orebro county, Sweden - proportion, characteristics and effective diagnostic solution in an emergent situation. Euro Surveill 2007;12:E5-6.

8 Herrmann B, Isaksson J, Ryberg M, et al. Global Multilocus Sequence Type Analysis of Chlamydia trachomatis Strains from 16 Countries. J Clin Microbiol 2015;53:2172-9.

9 Savage EJ, Ison CA, van de Laar MJ. Results of a Europe-wide investigation to assess the presence of a new variant of Chlamydia trachomatis. Euro Surveill 2007;12:E3-4.

10 Hoffmann S, Jensen JS. Mutant Chlamydia trachomatis in Denmark. Euro Surveill 2007;12:E7-8.

11 Niemi S, Hiltunen-Back E, Puolakkainen M. Chlamydia trachomatis genotypes and the Swedish new variant among urogenital Chlamydia trachomatis strains in Finland. Infect Dis Obstet Gynecol 2011;2011:1-6.

12 Shipitsyna E, Hadad R, Ryzhkova O, et al. First reported case of the Swedish new variant of Chlamydia trachomatis (nvCT) in Eastern Europe (Russia), and evaluation of Russian nucleic acid amplification tests regarding their ability to detect nvCT. Acta Derm Venereol 2012;92:330-1.

13 Fieser N, Simnacher U, Tausch Y, et al. Chlamydia trachomatis prevalence, genotype distribution and identification of the new Swedish variant in Southern Germany. Infection 2013;41:159-66.

14 Piñeiro L, Bernal S, Bordes A, et al. Minimum spread of the new swedish variant of Chlamydia trachomatis and distribution of $C$. trachomatis ompA genotypes in three geographically distant areas of Spain, 2011-2012. Infection 2014;42:905-12.

15 Nordvik MK, Liljeros F, Osterlund A, et al. Spatial bridges and the spread of Chlamydia: the case of a county in Sweden. Sex Transm Dis 2007:34:47-53.

16 Westh $\mathrm{H}$, Jensen JS. Low prevalence of the new variant of Chlamydia trachomatis in Denmark. Sex Transm Infect 2008;84:546-7.

17 Reinton N, Moi H, Bjerner J, et al. Den svenske chlamydiavarianten nvC trachomatis i Norge. Tidsskrift for Den norske legeforening 2010;130:380-1.

18 Herrmann B. A new genetic variant of Chlamydia trachomatis. Sex Transm Infect 2007;83:253-4.

19 Price MJ, Ades AE, Welton NJ, et al. Proportion of Pelvic Inflammatory Disease Cases Caused by Chlamydia trachomatis: Consistent Picture From Different Methods. J Infect Dis 2016;214:617-24.

20 Price MJ. Population Excess Fraction of Ectopic Pregnancy Due to Chlamydia trachomatis in Finland. Sex Transm Dis 2016;43:388-9.

21 Davies B, Turner KM, Frølund M, et al. Risk of reproductive complications following chlamydia testing: a population-based retrospective cohort study in Denmark. Lancet Infect Dis 2016;16:1057-64.

22 Low N, Egger M, Sterne JA, et al. Incidence of severe reproductive tract complications associated with diagnosed genital chlamydial infection: the Uppsala Women's Cohort Study. Sex Transm Infect 2006;82:212-8.

23 Price MJ, Ades AE, Soldan K, et al. The natural history of Chlamydia trachomatis infection in women: a multi-parameter evidence synthesis. Health Technol Assess 2016:20:1-250. 